\title{
Uso do azul de metileno na reversão de vasoplegia refratária ao uso de catecolaminas após bypass aortobifemoral
}

\author{
Use of methylene blue in the reversal of vasoplegia refractory to the use \\ of catecholamines after aortobifemoral bypass
}

\section{Mariana Raphaela Garcia de Araújo dos Santos, Luis Gustavo Campos da Silva, Paulo Roberto Barbosa Evora, Carlos Eli Piccinato, Edwaldo Edner Joviliano*}

\begin{abstract}
Resumo
Há evidências de que o óxido nítrico (NO) tem importância na vasodilatação associada a reações vasoplégicas. O objetivo deste relato de caso é documentar um caso de vasoplegia refratária ao uso de catecolaminas após bypass aortobifemoral revertida com o uso de azul de metileno. Mulher, 50 anos, submetida a bypass aortobifemoral. Sem comorbidades. Saiu de sala cirúrgica extubada, estável e com pulsos distais presentes. Duas horas após a cirurgia, evoluiu com choque circulatório. Iniciada noradrenalina e investigadas causas de choque. Manteve-se com necessidades crescentes de aminas e parâmetros estáveis. No sexto dia pós-operatório, com a hipótese de vasoplegia refratária, optou-se pelo uso do azul. Resposta imediata, com queda nos níveis de aminas, sendo desligada a noradrenalina no dia seguinte. $\mathrm{O}$ azul de metileno inibe a guanilato ciclase, produtora de guanosina monofosfato cíclico. Especula-se que haja um sinergismo entre essas drogas, já que a sua associação permite a atuação do sistema adenosina monofosfato.
\end{abstract}

Palavras-chave: Azul de metileno, vasoplegia, bypass aortobifemoral, óxido nítrico.

\section{Introdução}

Existem grandes evidências de que o óxido nítrico (NO) tem importância progressiva na vasodilatação associada a várias formas de choque circulatório. O NO é uma substância de vida curta, formada a partir do aminoácido L-arginina por um grupo de enzimas chamadas NO sintases (NOS), cuja produção normal é mantida por uma série de antígenos que atuam na superfície da célula endotelial, entre outras (Figura 1). O NO ativa a guanilil ciclase solúvel, diminuindo a quantidade de cálcio intracelular e facilitando o relaxamento do músculo liso. Atualmente, é conhecida a associação do $\mathrm{NO}$ a reações vasoplégicas relacionadas à sepse ${ }^{1-3} \mathrm{e}$ à anafilaxia ${ }^{4,5}$.

\begin{abstract}
There is evidence that nitric oxide plays an important role in the vasodilation associated with vasoplegic reactions. The objective of this case report is to document a case of catecholamine-refractory shock after aortobifemoral bypass treated with methylene blue. A 50-year-old woman was admitted for aortobifemoral bypass graft surgery. She did not present with any comorbidities. At the end of the surgery, she was breathing spontaneously, with palpable pedal pulses. Two hours later, the patient presented shock. Noradrenaline infusion was initiated and the causes of shock were investigated. She required increasing doses of amines and her parameters were stable. On the sixth day after surgery, methylene blue was administered due to the hypothesis of refractory vasoplegia. There was immediate response, with decrease in the catecholamine infusion levels until its withdrawal on the next day. Methylene blue inhibits the enzyme guanylate cyclase that produces GMPc. There might be a synergism between these two drugs, since their association allows the action of the AMPc system.
\end{abstract}

Keywords: Methylene blue, vasoplegia, aortobifemoral bypass, nitric oxide.

O objetivo deste relato de caso é documentar um caso de vasoplegia refratária ao uso de catecolaminas, provavelmente secundária a uma síndrome da resposta inflamatória sistêmica (SIRS), após bypass aortobifemoral que foi revertido com o uso de azul de metileno, um inibidor competitivo da enzima guanilil ciclase.

\section{Relato do caso}

Paciente do sexo feminino, 50 anos, foi submetida a um bypass aortobifemoral devido a obstrução aortoilíaca. Era tabagista por 30 anos e dislipidêmica. Não apresentava hipertensão arterial, cardiopatia ou diabetes melito. Medicações em uso: ácido acetilsalicílico e sinvastatina. Ao fi-

\footnotetext{
*Departamento de Cirurgia e Anatomia, Hospital das Clínicas de Ribeirão Preto (Faculdade de Medicina de Ribeirão Preto), Universidade de São Paulo (USP), Ribeirão Preto, SP.

Não foram declarados conflitos de interesse associados à publicação deste artigo.

Artigo submetido em 17.04.09, aceito em 19.10.09.

J Vasc Bras. 2010;9(1):74-77.

Copyright (C) 2010 by Sociedade Brasileira de Angiologia e de Cirurgia Vascular
} 


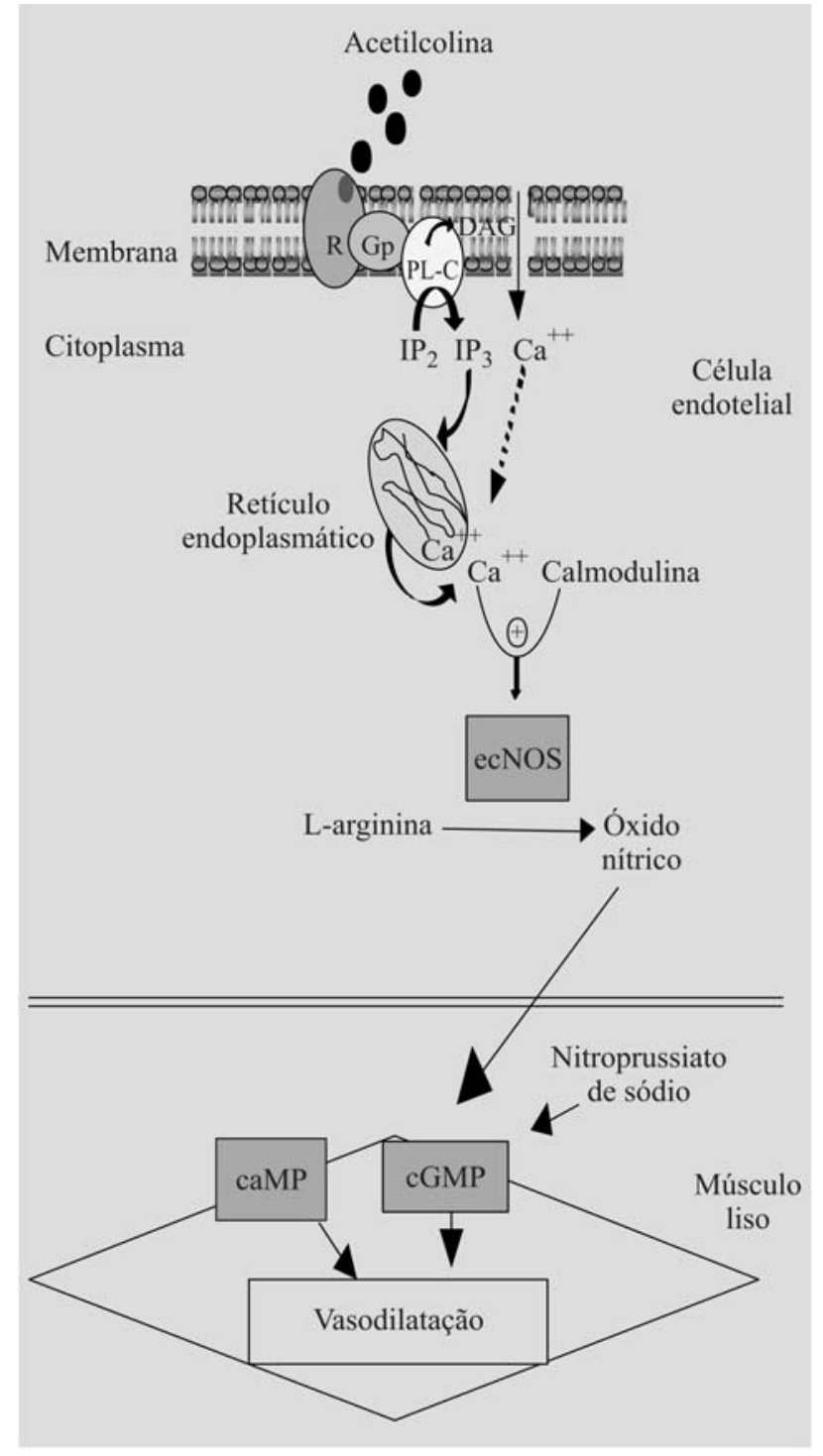

$\mathrm{AMPc}=$ adenosina monofosfato cíclico $; \mathrm{DAG}=$ diacilglicerol; ecNOS = óxido nítrico sintase endotelial constitutiva; $\mathrm{GMPc}=$ guanosina monofosfato cíclico; $\mathrm{Gp}=\mathrm{G}$-proteinas; $\mathrm{IP}_{3}=$ inositotrifosfato; $\mathrm{PIP}_{2}=$ fosfatidilinositol 4,5-bifosfato; $\mathrm{Pl}$ $\mathrm{C}=$ fosfolipase $\mathrm{C} ; \mathrm{R}=$ receptor de membrana

Figura 1 - Esquema da via de produção do óxido nítrico na célula endotelial e sua ação na célula muscular lisa através da indução do aumento da produção do guanosina monofosfato cíclico

nal da cirurgia, saiu da sala cirúrgica com respiração espontânea, consciente e orientada. Os pulsos distais antes ausentes estavam presentes e palpáveis com índice tornozelo-braquial (ITB) de 1,0.

Cerca de 2 horas após o final da cirurgia, a paciente evoluiu com hipotensão ( 60 x $40 \mathrm{~mm} \mathrm{Hg}$ ), frequência cardíaca (FC) de 100 bpm e pressão venosa central (PVC) de
$13 \mathrm{~cm} \mathrm{H}_{2} \mathrm{O}$. Iniciada noradrenalina a $0,5 \mathrm{~mL} / \mathrm{kg} / \mathrm{min}$. Foram investigadas causas para o choque hipovolêmico, cardiogênico e tromboembolismo pulmonar (acompanhamento dos níveis hematimétricos, raio $\mathrm{X}$ de tórax, ecocardiografia, além de todos os exames bioquímicos de rotina no pós-operatório de uma grande cirurgia). Todos os exames complementares estavam normais, com exceção de uma leucocitose (contagem de glóbulos brancos = 13.000 Gpt/L). Nas horas subsequentes, a paciente manteve-se com necessidades crescentes de aminas vasoativas para manter parâmetros hemodinâmicos.

No terceiro dia de pós-operatório, com todos os parâmetros estáveis (diurese, sem queda nos valores hematimétricos), apresentou um pico febril de $38^{\circ} \mathrm{C}$.

No sexto dia de pós-operatório, deparamos-nos com a seguinte situação: noradrenalina: $2,4 \mathrm{mcg} / \mathrm{kg} / \mathrm{min}$, dopamina: $20 \mathrm{mcg} / \mathrm{min}$, sem alterações hematimétricas, PVC: $18 \mathrm{~cm} \mathrm{H}_{2} 0$, diurese: $100 \mathrm{~mL} /$ hora, PA: 70 x 50, FC: $120 \mathrm{bpm}$, paciente aquecida e com pulsos em membros inferiores palpáveis e amplos.

Com a hipótese diagnóstica de vasoplegia refratária ao uso de aminas, provavelmente secundária a SIRS, optou-se pelo uso do azul de metileno $(2 \mathrm{~mL} / \mathrm{kg}$ em bolus EV) e, após 2 horas, a mesma dose. Durante o bolus de azul de metileno, a noradrenalina foi reduzida para $1,5 \mathrm{mcg} / \mathrm{kg} / \mathrm{min}$, sendo desligada a dopamina. No dia seguinte, a dose de noradrenalina era de $0,25 \mathrm{mcg} / \mathrm{kg} / \mathrm{min}$. No sétimo dia de pós-operatório foi suspenso o uso de noradrenalina. A paciente permaneceu internada no Centro de Terapia Intensiva (CTI), pois necessitou de suporte ventilatório.

Teve alta para enfermaria no $23^{\circ}$ dia pós-operatório, já que foi necessário finalizar tratamento para pneumonia. Apresentava-se afebril, estável, sem alterações de ferida operatória, aceitando dieta com pulsos presentes e amplos $($ ITB $=1)$. Alta hospitalar no $26^{\circ}$ dia de pós-operatório.

Retornou ao nosso ambulatório após 30 dias sentindo-se bem, sem queixas, deambulando com ITB $=1,0$ bilateralmente. Sem alterações de ferida operatória. Foi remarcado retorno em 3 meses para reavaliação clínica.

\section{Discussão}

A síndrome da resposta inflamatória sistêmica é desencadeada pela produção de cininas pró-inflamatórias e pode resultar em disfunção orgânica nos casos mais gra- 
ves. É mais frequentemente observada em pós-operatórios de cirurgia cardíaca com perfusão extracorpórea, podendo levar a lesão de reperfusão miocárdica, lesão pulmonar e profunda vasodilatação sistêmica, aumentando muito a morbidade pós-operatória. A SIRS é definida, segundo o American College of Chest Physicians / Society of Critical Care Medicine Consensus Conference, pela ocorrência de dois ou mais dos seguintes sinais: anormalidades da temperatura corporal $\left(>38^{\circ} \mathrm{C}<36{ }^{\circ} \mathrm{C}\right)$; taquicardia persistente $(\mathrm{FC}>90 \mathrm{bpm})$; taquipneia ou hiperventilação (frequência respiratória $>20 / \mathrm{min}$, ou pressão parcial de $\mathrm{CO}_{2}<32 \mathrm{~mm} \mathrm{Hg}$ ) e leucocitose ou leucopenia (contagem de leucócitos $>12.000 \mathrm{Gpt} / \mathrm{L}$ ou $<4.000 \mathrm{Gpt} / \mathrm{L})^{6}$. O mecanismo da SIRS não é totalmente compreendido. A ativação de neutrófilos e radicais livres, endotoxinas e produção de cininas são mecanismos participantes no processo inflamatório da SIRS assim como ocorre nos processos de lesão na isquemia-reperfusão músculo-esquelética ${ }^{5,7,8}$. A vasoplegia observada no caso descrito teve comportamento semelhante à observada nos casos descritos pós-cirurgia cardíaca. Winnock et al. já descreveram estado de colapso circulatório com marcante eritema cutâneo lembrando o choque anafilático após cirurgia aortoilíaca ${ }^{6}$.

O mecanismo pelo qual se estabelece a vasoplegia ocorre como nas reações adversas observadas quando do uso da protamina ou na sepse. A indução, através de antígenos específicos, de receptores de superfície dos mastócitos desencadeia a liberação de uma série de mediadores, alguns deles levando ao aumento da produção de mediadores e alguns deles levando ao aumento da produção de NO através da regulação da NOS. Outra possibilidade é a de que outros antígenos possam estimular diretamente a célula endotelial na conversão do aminoácido L-arginina a $\mathrm{NO}^{9}$.

A síntese de NO pelo organismo é regulada pelas NOS, que se dividem em 3 tipos: NOS neuronal, NOS constitutiva e NOS induzível (Figura 2). Em situações de sepse, anafilaxia e síndromes vasoplégicas, ocorre uma ativação das NOS induzíveis, aumentando de forma exponencial a quantidade de NO no organismo. Esse aumento exacerbado faz com que haja um relaxamento da musculatura lisa dependente da guanosina monofosfato (GMPc). Este mecanismo tem uma íntima correlação com o sistema adenosina monofosfato (AMPc), no qual age a noradrena-

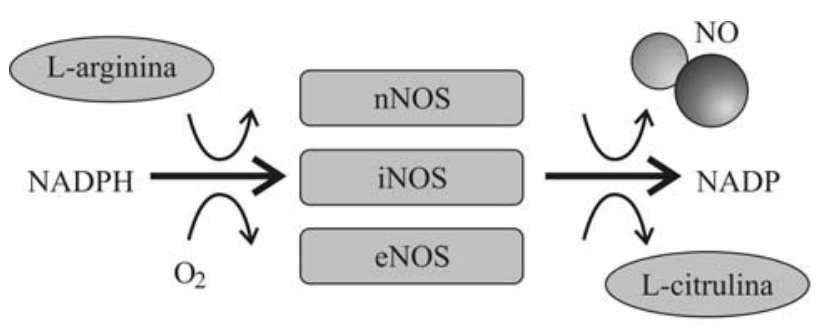

eNOS = óxido nítrico sintase constitutiva; $i N O S=$ óxido nítrico sintase induzivel; NADP = nicotinamida adenosina dinucleotídeo fosfato forma reduzida; NADPH = nicotinamida adenosina dinucleotídeo fosfato forma oxidada; $\mathrm{nNOS}=$ óxido nítrico sintase neuronal.

Figura 2 - Representação da produção do óxido nítrico pelas enzimas óxido nítrico sintases (nNOS, iNOS e eNOS) a partir do substrato principal L-arginina

lina (feedback negativo). A via do GMPc, quando muito ativada, bloqueia a via do AMPc (autorregulação).

O uso do azul de metileno tem sido descrito em diferentes situações clínicas. Sua ação na meta-hemoglobinemia já foi descrita, podendo ser usado no tratamento crônico da urolitíase. Mais recentemente, o seu uso tem sido descrito no tratamento das alterações hemodinâmicas da vasoplegia refratária, na anafilaxia e no choque séptico ${ }^{10}$. Sua toxicidade relacionada à dose inclui anemia hemolítica, arritmias cardíacas, descamação de pele, dor abdominal, pele e urina azuladas, além de produzir hiperbilirrubinemia em neonatos. Sua ação consiste na inibição competitiva da enzima guanilato ciclase, produtora do GMPc. Essa inibição do sistema GMPc permitiria uma melhor atuação do sistema AMPc induzido pela noradrenalina. No caso descrito ressaltamos a ocorrência do fenômeno vasoplégico após cirurgia aortoilíaca, provavelmente secundário a SIRS desencadeada pelo pinçamento aórtico e a reperfusão. Deve ser enfatizado que a noradrenalina continua sendo a droga de escolha em situações de choque vasoplégico. O que se pode especular é o sinergismo entre essas duas drogas, pois a sua associação permite que o sistema AMPc fique livre para atuar, já que há um bloqueio, pelo menos parcial, no sistema GMPc pela ação do azul de metileno (cross-talk).

\section{Referências}

1. Kilbourn RG, Traber DL, Szabo C. Nitric oxide and shock. Dis Mon.1997;43:277-348.

2. Evora PRB, Seccombe JF, Pearson PJ, Schaff HV. O papel do óxido nítrico e perspectivas terapêuticas na sepse bacteriana. Ver Bras Terap Intens. 1994;6:105-7. 
3. Schneider F. Methylene blue infusion in septic shock. Crit Care Med. 1995;23:1935-1936.

4. Osada S, Ichiki H, Oku H, Ishiguro K, Kunitomo M, Semma M. Participation of nitric oxide in mouse anaphylactic hypotension. Eur J Pharmacol. 1994;252:347-50.

5. Wan S, Leclerc JL, Vincent JL. Inflammatory response to cardiopulmonary bypass: Mechanisms involved and possible therapeutic strategies. Chest. 1997;112:676-92.

6. Winnock S, Janvier G, Cesarini M, et al. Hemodinamic changes caused by laparotomy during aorto-iliac surgery. Ann Fr Reanim. 1987;6:471-5.

7. Seghaye MC, Duchateau J,Grabitz GR, et al. Complement activation during cardiopulmonary bypass in infants and children: relation to postoperative multiple system organ failure. J Thorac Cardiovasc Surg. 1993;106:978-87.

8. Patrick DA, Moore EE, Fullerton DA, et al. Cardiopulmonary bypass renders patients at risk for multiple organ failure via early neutrophil priming and late neutrophil disability. J Surg Res. 1999;86:42-9.

9. Pearson PJ, Evora PRB, Ayrancioglu K, Schaff HV. Protamine releases endothelium-derived relaxing factor from systemic arteries: a possible mechanism of hypotension duryng heparin neutralization. Circulation. 1992;86;289-94.
10. Evora PRB, Roselino CHCD, Schiaveto PM. Methylene blue in anaphylatic shock. Ann Emeg Med. 1997;30:240.

Correspondência:

Mariana Raphaela Garcia de Araújo dos Santos

Avenida do Café, 1825/33

CEP 14050-230 - Ribeirão Preto, SP

e-mail: marianargasantos@hotmail.com

\section{Contribuições dos autores}

Concepção e desenho do estudo: MRGAS, EEJ

Análise e interpretação dos dados: MRGAS, LGCS, EEJ

Coleta de dados: MRGAS, LGCS

Redação do artigo: MRGAS, LGCS, EEJ

Revisão crítica do texto: MRGAS, LGCS, EEJ, PRBE, CEP

Aprovação final do artigo*: MRGAS, LGCS, PRBE, CEP, EEJ

Análise estatística: N/A

Responsabilidade geral pelo estudo: MRGAS, EEJ

Informações sobre financiamento: N/A

* Todos os autores leram e aprovaram a versão final submetida ao J Vasc Bras. 